\title{
Rhinovirus is the most common virus and rhinovirus- $C$ is the most common species in paediatric intensive care respiratory admissions
}

To the Editor:

Acute respiratory illnesses (ARIs) account for $10-15 \%$ of all admissions to the paediatric intensive care unit (PICU) [1]. Historically, respiratory syncytial virus was reported to be the most common viral pathogen resulting in admission to the PICU [2]. Recent studies, using more sensitive molecular techniques, have shown that rhinoviruses may be the most frequent virus detected in respiratory admissions to a PICU [3-5].

We have previously reported that the rhinovirus- $C$ species is associated with more frequent respiratory admissions $[6,7]$. There has been no study reported to date examining the prevalence of each rhinovirus species in children admitted with an ARI to the PICU.

The aim of this study was to determine the prevalence of different viruses, in particular rhinovirus species, in children admitted to a PICU with an ARI.

Patient information was obtained retrospectively from the hospital computer database as part of an audit on all children admitted with an ARI to the PICU at Princess Margaret Hospital (PMH), Perth, Australia, between March 2009 and July 2011.

We compared these data collected with those from a concurrent prospective study running at $\mathrm{PMH}$ examining the mechanisms of acute viral respiratory infections in children (MAVRIC) [7]. The MAVRIC study enrolled children presenting to the emergency department with acute asthma, bronchiolitis and pneumonia. We compared our PICU cohort with all cases with the above ARIs not requiring PICU admission that were recruited to the MAVRIC study over the same timeframe.

Detection of common respiratory viruses from nasopharyngeal aspirate (NPA) samples were completed as previously described $[6,8-10]$.

From March 2009 to July 2011, 260 children, in total, were admitted to the PICU with an ARI. Of this cohort, 229 (88.1\%) had an NPA performed. The mean \pm SD age was $2.8 \pm 4.0$ (range $0-16$ ) years and the most common respiratory diagnoses were acute asthma $(n=63 ; 27.5 \%)$, bronchiolitis $(n=67 ; 29.3 \%)$ and pneumonia $(\mathrm{n}=44 ; 19.2 \%)$.

Rhinovirus was the most frequent virus detected; being present in 94 (41.0\%) of the NPA samples. respiratory syncytial virus was identified in 50 (21.8\%) samples.

Based on the analysis of prevalence of rhinovirus species, rhinovirus- $\mathrm{C}$ was the most common rhinovirus species detected, being present in $51(22.3 \%)$ of the 229 samples (figure 1). Rhinovirus-A species was found in $40(17.5 \%)$ and rhinovirus-B in $4(1.7 \%)$ of the overall PICU cohort.

Of the overall 260 patients admitted to PICU with an ARI, 31 (11.9\%) did not have an NPA taken. The demographics of these patients were compared with those PICU respiratory admissions that had an NPA

@ERSpublications

This is first report to examine the role of different RV species in ARIs in children admitted to paediatric ICU. Our study found that RV-C is the most common RV species in paediatric intensive care respiratory admissions. http://ow.ly/P6EN30k9UnX

Cite this article as: Cox DW, Khoo S-K, Zhang G, et al. Rhinovirus is the most common virus and rhinovirus- $\mathrm{C}$ is the most common species in paediatric intensive care respiratory admissions. Eur Respir $J$ 2018; 52: 1800207 [https://doi.org/10.1183/13993003.00207-2018]. 
FIGURE 1 Rhinovirus species (HRV) detected compared with respiratory syncytial virus (RSV) and other viruses in the paediatric intensive care unit group. NPA: nasopharyngeal aspirate.

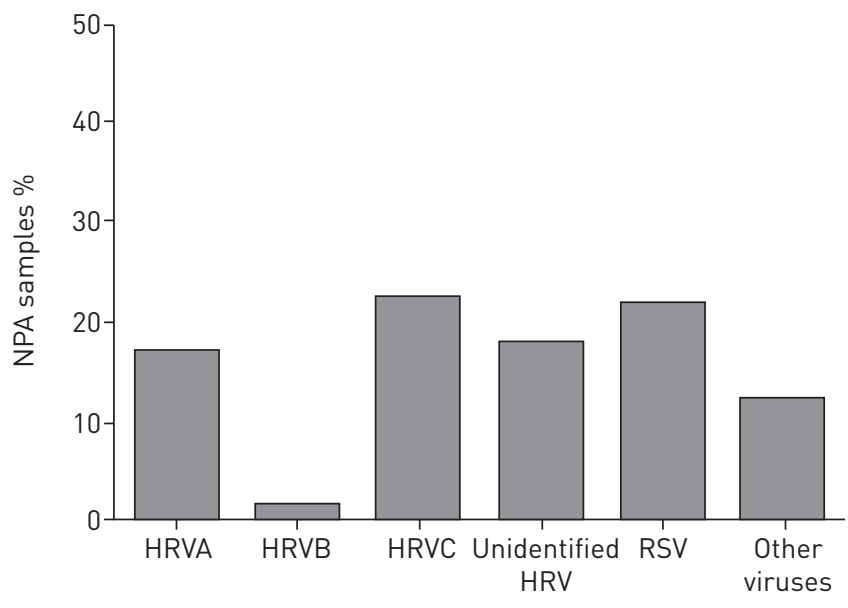

Virus detected

taken. The children who did not have an NPA performed were older $(6.41 \pm 5.34$ versus $2.8 \pm 4.0$ years; $\mathrm{p}<0.001)$ and more likely to have a diagnosis of pneumonia $(35.5 \%$ versus $19.2 \%$; $=0.01)$.

Over the duration of this study, 181 emergency department ARI cases were recruited to the MAVRIC study and these subjects were compared with the cohort admitted to the PICU. Patients admitted to the PICU were more likely to stay in hospital longer $(23.7 \pm 54.5$ versus $1.4 \pm 1.39$ days; $\mathrm{p}<0.001)$ and have a co-morbidity $(\mathrm{n}=88,38.4 \%$ versus $\mathrm{n}=0 ; \mathrm{p}<0.001)$ compared with emergency department ARI cases admitted to the ward.

Subjects admitted to the PICU versus emergency department ARI cases were less likely to have a diagnosis of asthma $(27.5 \%$ versus $66.8 \%$, respectively; $\mathrm{p}<0.001)$ but more likely to have a diagnosis of pneumonia ( $19.6 \%$ versus $5.6 \%$, respectively; $\mathrm{p}<0.001)$. Rhinovirus-C was the most common rhinovirus species detected in both sets of hospital cases. There was no statistical difference identified between the two groups with any particular virus.

Of the 229 children admitted to the PICU who had NPA, 24 (10.5\%) had a co-infection with another virus. Comparing clinical outcomes of the children who had a co-infection with the children with one virus detected, there was no statistically significant difference between the two groups.

This study shows that rhinovirus is the most common virus identified in children admitted to a PICU with an ARI and rhinovirus-C is the most frequent rhinovirus species detected in children positive for rhinovirus. Importantly, our data show that rhinovirus- $\mathrm{C}$ is a common viral pathogen detected in PICU ARI admissions and as common, or perhaps even more common than respiratory syncytial virus.

This is the first study to systematically examine relationships between respiratory viruses and different rhinovirus species in children admitted to a PICU with an ARI and, to our knowledge, is the largest study in a PICU population using RT-PCR rhinovirus molecular typing methods. Previous studies either did not include rhinovirus typing and used older virological detection methods [2] or were too small to report specifically on the prevalence of rhinovirus species [3, 4].

Our research group has previously demonstrated that rhinovirus-C-related wheezing episodes in preschool children are associated with an increased risk of previous and subsequent respiratory hospital admissions compared with other rhinovirus species [7]. Recent experimental evidence established that rhinovirus-C species has the ability to grow equally well at both $34^{\circ} \mathrm{C}$ and $37^{\circ} \mathrm{C}$ [11] which helps to explain why rhinovirus- $\mathrm{C}$ species are frequently found in severe lower respiratory illnesses in children.

Major strengths in this study include the use of up-to-date, highly sensitive PCR techniques and that it included a number of different respiratory diagnoses over different seasons. The findings in our study are strengthened by the inclusion of a control group. Although there were some differences in the clinial demographics between the two groups reported, the MAVRIC ARI cases provide corroborating evidence on the frequency of different viruses in children admitted to the PICU with an ARI.

In this study, viral detection data was not available on 31 (11.9\%) of the overall PICU respiratory admissions. These children were older and more likely to have a diagnosis of pneumonia. NPAs are not routinely performed on older children given the level of discomfort associated with the procedure. Both the PICU and emergency department ARI cohorts where an NPA was performed were mainly younger pre-school children admitted with either bronchiolitis or asthma. Previous studies have demonstrated a 
similar frequency and distribution of rhinovirus infections in preschool children with wheezing disorders verifying our findings [7, 12-15]. A subsection analysis was required to determine rhinovirus species as 91 (40\%) of the collected NPAs were no longer available for rhinovirus typing. Given the size of this sub-analysis and that the availability of these specimens was random, the data reported could be expected to represent the profile of the distribution of different rhinovirus species in children admitted to the PICU with an ARI.

In conclusion, this is the first report examining the role of different rhinovirus species in ARIs in children admitted to a tertiary PICU. Rhinovirus was the most common virus detected and rhinovirus-C was the most prevalent rhinovirus species detected. Rhinovirus- $\mathrm{C}$ was also the most common rhinovirus species detected in children admitted to the PICU with a diagnosis of either acute asthma or bronchiolitis. Importantly, rhinovirus- $\mathrm{C}$ was as commonly detected in PICU admissions as respiratory syncytial virus.

Desmond W. $\operatorname{Cox}^{1,2}$, Siew-Kim Khoo ${ }^{2,3}$, Guicheng Zhang ${ }^{2,4}$, Katie Lindsay ${ }^{5}$, Anthony D. Keil ${ }^{5}$, Geoff Knight ${ }^{6}$, James E. Gern ${ }^{7}$, Ingrid A. Laing ${ }^{2,3}$, Joelene Bizzintino ${ }^{2,3}$ and Peter N. Le Souëf ${ }^{2}$

${ }^{1}$ Respiratory Dept, Our Lady's Children's Hospital, Dublin, Ireland. ${ }^{2}$ School of Paediatrics and Child Health, University of Western Australia, Perth, Australia. ${ }^{3}$ Telethon Kids Institute, University of Western Australia, Perth, Australia ${ }^{4}$ School of Public Health, Curtin University, Centre for Genetic Origins of Health and Disease, Curtin University and the University of Western Australia, Perth, Australia. ${ }^{5}$ Microbiology Dept, PathWest Laboratory Medicine, Perth, Australia. ${ }^{6}$ Paediatric Intensive Care, Princess Margaret Hospital, Perth, Australia. ${ }^{7}$ Dept of Paediatrics, University of Wisconsin-Madison, Madison, WI, USA.

Correspondence: D.W. Cox, Respiratory Dept, Our Lady’s Children's Hospital, Crumlin, Dublin, Ireland.

E-mail: des.cox@olchc.ie

Received: Jan 312018 | Accepted after revision: May 132018

Conflict of interest: P.N. Le Souëf reports grants from National Health and Medical Research Council Australia (programme grant number APP458513, project grant number APP1031635), during the conduct of the study.

Support statement: This study was supported by National Health and Medical Research Council (NHMRC) funding. Funding information for this article has been deposited with the Crossref Funder Registry.

\section{References}

1 Purcell K, Fergie J. Driscoll Children's Hospital respiratory syncytial virus database: risk factors, treatment and hospital course in 3308 infants and young children, 1991 to 2002. Pediatr Infect Dis J 2004; 23: 418-423.

2 Straliotto SM, Siqueira MM, Machado V, et al. Respiratory viruses in the pediatric intensive care unit: prevalence and clinical aspects. Mem Inst Oswaldo Cruz 2004; 99: 883-887.

3 Louie JK, Roy-Burman A, Guardia-Labar L, et al. Rhinovirus associated with severe lower respiratory tract infections in children. Pediatr Infect Dis J 2009; 28: 337-339.

4 Aramburo A, van Schaik S, Louie J, et al. Role of real-time reverse transcription polymerase chain reaction for detection of respiratory viruses in critically ill children with respiratory disease: is it time for a change in algorithm? Pediatr Crit Care Med 2011; 12: e160-e165.

5 Lonngren C, Morrow BM, Haynes S, et al. North-South divide: distribution and outcome of respiratory viral infections in paediatric intensive care units in Cape Town (South Africa) and Nottingham (United Kingdom). J Paediatr Child Health 2014; 50: 208-215.

6 Bizzintino J, Lee WM, Laing IA, et al. Association between human rhinovirus C and severity of acute asthma in children. Eur Respir J 2011; 37: 1037-1042.

7 Cox DW, Bizzintino J, Ferrari G, et al. Human rhinovirus species C infection in young children with acute wheeze is associated with increased acute respiratory hospital admissions. Am J Respir Crit Care Med 2013; 188: $1358-1364$.

8 Lee WM, Grindle K, Pappas T, et al. High-throughput, sensitive, and accurate multiplex PCR-microsphere flow cytometry system for large-scale comprehensive detection of respiratory viruses. J Clin Microbiol 2007; 45: 2626-2634.

9 Lee WM, Kiesner C, Pappas T, et al. A diverse group of previously unrecognized human rhinoviruses are common causes of respiratory illnesses in infants. PLoS One 2007; 2: e966.

10 Bochkov YA, Grindle K, Vang F, et al. Improved molecular typing assay for rhinovirus species A, B, and C. J Clin Microbiol 2014; 52: 2461-2471.

11 Ashraf S, Brockman-Schneider R, Bochkov YA, et al. Biological characteristics and propagation of human rhinovirus-C in differentiated sinus epithelial cells. Virology 2013; 436: 143-149.

12 Miller EK, Edwards KM, Weinberg GA, et al. A novel group of rhinoviruses is associated with asthma hospitalizations. J Allergy Clin Immunol 2009; 123: 98-104 e1.

13 Miller EK, Khuri-Bulos N, Williams JV, et al. Human rhinovirus C associated with wheezing in hospitalised children in the Middle East. J Clin Virol 2009; 46: 85-89.

14 Iwane MK, Prill MM, Lu X, et al. Human rhinovirus species associated with hospitalizations for acute respiratory illness in young US children. J Infect Dis 2011; 204: 1702-1710.

15 Khetsuriani N, et al. Novel human rhinoviruses and exacerbation of asthma in children. Emerg Infect Dis 2008; 14 1793-1796. 